\title{
NEW STABILITY CRITERIA FOR REACTION-DIFFUSION
}

\author{
M.A. BIOT \\ Royal Academy of Belgium, Brussels, Belgium
}

Received 30 August 1984

\begin{abstract}
New stability criteria are developed for steady state evolution of reaction-diffusion far from equilibrium. The derivation is based on the lagrangian equations of irreversible thermodynamics. A new and general expression is also developed for the affinity which embodies the chemical kinetics.
\end{abstract}

\section{Introduction}

A new variational approach to irreversible thermodynamics has been developed leading to lagrangian equations of evolution. The essential results have been presented recently in the form of a short monograph [1]. Its foundation rests on a fundamental principle of virtual dissipation.

Our purpose here is to derive new stability criteria for a steady state evolution based on lagrangian equations in the context of general reaction-diffusion. As a preliminary, the concepts of local energy and entropy per unit volume under irreversible evolution are given a precise definition and a new expression is also developed for the affinity which embodies chemical kinetics. A special derivation of the variational and the lagrangian equations is presented in the particular context of reaction-diffusion as well as a general expression for thermomolecular dissipation which is invariant under translation.

A new stability criterion is obtained which does not require "local equilibrium" and distinguishes between oscillatory and non-oscillatory stability. The lagrangian equations also lead directly to a stability criterion analogous to that proposed by Glansdorff and Prigogine [2] providing at the same time a precise definition of an associated condition of "local equilibrium".

\section{Outline of a new thermochemistry}

We shall briefly recall the new viewpoints and results introduced earlier in the analysis of open chemically reacting systems, and based on concepts which differ fundamentally from standard procedures [1].

Consider a rigid cell $\mathrm{C}_{\mathrm{P}}$ containing a homogeneous mixture of pure substances $k$ in equilibrium at a given temperature. It is subject to a transformation which brings it to a certain temperature $T$ while masses $M^{k}$ are added to it from outside. At the same time masses $\nu_{k} \xi$ are also added to it by a chemical reaction of coordinate $\xi$. We have shown that the same transformation may be obtained reversibly by a process called thermobaric transfer.

We refer to $C_{P}$ as the primary cell and adjoin to it large rigid supply cells $\mathrm{C}_{k}$ each containing the substance $k$ at the same pressure and temperature $p_{0} T_{0}$. We also adjoin a chemical supply cell $\mathrm{C}_{\mathrm{ch}}$ containing the reactants in chemical equilibrium along with a thermal well $\mathrm{TW}$ which is a large rigid reservoir at constant temperature $T_{0}$. The system $\mathrm{C}_{\mathrm{P}}+\mathrm{C}_{\mathrm{ch}}+\sum_{k} \mathrm{C}_{k}+\mathrm{TW}$ is called a hypersystem.

In order to illustrate the process of thermobaric transfer we extract a mass $\mathrm{d} M^{k}$ from the supply cell, bring it reversibly to a state of equilibrium with the primary cell at the pressure $p_{k}$ and temperature $T$ and inject it isothermally and reversibly into it through a semipermeable membrane. The 
increase of energy of the system $\mathrm{C}_{\mathrm{P}}+\sum_{k} \mathrm{C}_{k}$ is

$\mathrm{d} \mathscr{U}^{M}=\sum_{k}\left(\bar{\epsilon}_{k}+h_{v T}^{k}\right) \mathrm{d} M^{k}$,

with

$\bar{\epsilon}_{k}=\int_{p_{0} T_{0}}^{p_{k} T}\left(\mathrm{~d} p_{k}^{\prime} / \rho_{k}^{\prime}+T^{\prime} \mathrm{d} \bar{s}_{k}\right)$.

We have called $\bar{\epsilon}_{k}$ the injection enthalpy and $p_{k}$ the injection pressure and denote by $p_{k}^{\prime}, \rho_{k}^{\prime}, T^{\prime}$ and $\bar{s}_{k}$ the pressure, density, temperature, and specific entropy of $\mathrm{d} M^{k}$ along the path of integration, respectively. The term $h_{v T}^{k} \mathrm{~d} M^{k}$ represents the heat to be added to $C_{P}$ in order to maintain a constant temperature during injection.

We may also consider a reaction $\mathrm{d} \xi$ to occur in the chemical cell $\mathrm{C}_{\mathrm{ch}}$ at constant temperature $T_{\text {eq }}$ which is maintained by injecting into it an amount of heat $\bar{h}_{T}^{\text {eq }} \mathrm{d} \xi$. The masses $\nu_{k} \mathrm{~d} \xi$ produced are extracted from $\mathrm{C}_{\mathrm{ch}}$ and injected by thermobaric transfer into $C_{P}$. Finally the temperature of $C_{P}$ is increased by an amount $\mathrm{d} T$.

Thus we end up with a system whose final state is the same as if masses $\mathrm{d} M^{k}$ had been transferred into the primary cell while a chemical reaction $d \xi$ takes place in it with an increase of temperature $\mathrm{d} T$.

The increase of energy of the system $\mathrm{C}_{\mathrm{P}}+\mathrm{C}_{\mathrm{ch}}$ $+\sum_{k} \mathrm{C}_{k}$ in this combined process is

$$
\begin{aligned}
\mathrm{d} \mathscr{U}= & \sum_{k}\left(\bar{\epsilon}_{k}+h_{v T}^{k}\right) \mathrm{d} M^{k}+\sum_{k}\left(\bar{\epsilon}_{k}-\bar{\epsilon}_{k}^{\mathrm{ch}}+h_{v T}^{k}\right) \nu_{k} \mathrm{~d} \xi \\
& +\bar{h}_{T}^{\mathrm{eu}} \mathrm{d} \xi+C_{v m} \mathrm{~d} T .
\end{aligned}
$$

In this expression $\bar{\epsilon}_{k}^{\mathrm{ch}}$ is the injection enthalpy in $\mathrm{C}_{\mathrm{ch}}$. Similarly we obtain the increase of entropy of the system $\mathrm{C}_{\mathrm{P}}+\mathrm{C}_{\mathrm{ch}}+\sum_{k} \mathrm{C}_{k}$ as

$$
\begin{aligned}
\mathrm{d} \mathscr{S}= & \sum_{k}\left(\bar{s}_{k}+h_{v T}^{k} / T\right) \mathrm{d} M^{k} \\
& +\sum_{k}\left(\bar{s}_{k}-\bar{s}_{k}^{\mathrm{ch}}+h_{v T}^{k} / T\right) \nu_{k} \mathrm{~d} \xi \\
& +\left(\bar{h}^{\mathrm{eq}} / T_{\text {eq }}\right) \mathrm{d} \xi+\left(C_{v m} / T\right) \mathrm{d} T,
\end{aligned}
$$

where

$\bar{s}_{k}=\int_{p_{0} T_{0}}^{p_{k} T} \mathrm{~d} \bar{s}_{k}$ is the injection entropy and $\bar{s}_{k}^{\text {ch }}$ its value for the chemical cell.

The quantities $\bar{\epsilon}_{k}, \bar{s}_{k}, h_{v T}^{k}$ and $C_{v m}$ appearing in the coefficients of the differentials (2.3) and (2.4) may be determined as functions of the temperature and composition of $\mathrm{C}_{\mathrm{P}}$ hence as functions of $T$ and $M^{k}+\nu_{k} \xi$. By integration they yield

$\mathscr{U}=\mathscr{U}\left(M^{k}, \xi, T\right), \mathscr{S}=\mathscr{P}\left(M^{k}, \xi, T\right)$,

as function of $M^{k} \xi$ and $T$. These values may be considered as defining the energy and entropy of the primary cell although they involve the combined system $\mathrm{C}_{\mathrm{P}}+\mathrm{C}_{\mathrm{ch}}+\sum_{k} \mathrm{C}_{k}$. As a consequence it is interesting to note that they depend not only on the composition $M^{k}+v_{k} \xi$ of the cell but also on $M^{k}$ and $\xi$ as separate independent variables.

After multiplying (2.4) by $T$ and subtracting it from (2.3) we obtain

$\mathrm{d} \mathscr{U}=-A \mathrm{~d} \xi+\sum_{k} \phi_{k} \mathrm{~d} M^{k}+T \mathrm{~d} \mathscr{S}$,

where

$\phi_{k}=\bar{\epsilon}_{k}-T \bar{s}_{k}$

was called the convective potential. The quantity $A$ is

$A=\sum_{k}\left[T\left(\bar{s}_{k}-\bar{s}_{k}^{\mathrm{ch}}\right)-\bar{\epsilon}_{k}+\bar{\epsilon}_{k}^{\mathrm{ch}}\right] \nu_{k}+\bar{h}_{T}^{\mathrm{eq}}\left(T / T_{\mathrm{eq}}-1\right)$.

It represents the affinity since for an isolated system $\left(\mathrm{d} \mathscr{U}=\mathrm{d} M^{k}=0\right)$ eq. (2.7) yields

$A=T \mathrm{~d} \mathscr{S}$.

Eq. (2.10) is a new form of Gibbs' equation which avoids the use of the chemical potential. Thereby bypassing the difficulty due to the presence of undetermined constants in this potential.

When there are simultaneous chemical reactions of coordinates $\xi_{\rho}$ eqs. (2.3) and (2.4) are readily generalized to this case by adjoining to the hypersystem chemical cells $\mathrm{C}_{\mathrm{ch} \rho}$ for each reaction. Eq. (2.7) is thus generalized to

$\mathrm{d} \mathscr{U}=-\sum_{\rho} A_{\rho} \mathrm{d} \xi_{\rho}+\sum_{k} \phi_{k} \mathrm{~d} M^{k}+T \mathrm{~d} \mathscr{S}$,

while expressions (2.6) become

$\mathscr{U}=\mathscr{U}\left(M^{k}, \xi_{\rho}, T\right), \quad \mathscr{S}=\mathscr{S}\left(M^{k}, \xi_{\rho}, T\right)$. 


\section{Local energy and entropy of a continuous irreversible system}

The energy and entropy of an open rigid cell have been defined above by a reversible process called thermobaric transfer. The volume of the cell is arbitrary and may be taken as unity. In that case $\mathscr{U}$ and $\mathscr{S}$ are the values of the energy and entropy per unit volume. The variables are now the temperature $T$ and the masses $M^{k}$ added by convection per unit volume while $\xi$ defines the masses $\nu_{k} \xi$ produced by the chemical reaction also per unit volume. If we consider a continuous system in irreversible evolution occupying a domain $\Omega$ the energy and entropy of an elementary domain $\mathrm{d} \Omega$ are respectively $\mathscr{U} \mathrm{d} \Omega$ and $\mathscr{S} \mathrm{d} \Omega$ where $\mathscr{U}$ and $\mathscr{S}$ are functions of the instantaneous local values of $T$ and of $M^{k}$ and $\xi$ per unit volume. The total energy $U$ and entropy $S$ of the domain $\Omega$ are then expressed by the volume integrals

$$
U=\int_{\Omega} \mathscr{U} \mathrm{d} \Omega, \quad S=\int_{\Omega} \mathscr{P} \mathrm{d} \Omega .
$$

Underlying this approach is the assumption that the domain may be divided into elements which are sufficiently small to be treated as infinitesimal while their actual size remains above a resolution threshold for which $T, M^{k}$ and $\xi$ retain their physical significance as defined above with reference to a unit volume. The local values of $\phi_{k}$ and $A_{\rho}$ are intensive variables functions of the temperature $T$ and

$m_{k}=m_{0 k}+\sum_{\rho} \nu_{k \rho} \xi_{\rho}+M^{k}$,

where $m_{k}$ is the mass of substance $k$ per unit volume, $m_{0 k}$ being its initial value, while $\nu_{k \rho} \xi_{\rho}$ and $M^{k}$ are the values added by chemical reaction and convection. Note that $m_{k}$ may represent all the masses present in the system per unit volume or only those substances which participate in convection and reaction as solutes in a rigid neutral support.

\section{Entropy production and dissipation forces}

In an irreversible process entropy is produced. It may be evaluated in several ways. In the present context of reaction-diffusion one of the simplest is the following.

We consider the mass flux $\dot{M}_{i}^{k}$ of substance $k$ per unit area. If we denote by $\dot{H}_{i}$ the heat flux, the diffusive energy flux is (the dot indicates a time derivative)

$\dot{\mathscr{F}}_{i}=\sum_{k} \bar{\epsilon}_{k} \dot{M}_{i}^{k}+\dot{H}_{i}$.

We denote by $\mathscr{F}_{i} M_{i}^{k}$ and $H_{i}$ the time integrals of $\dot{\mathscr{F}}_{i} \dot{M}_{i}^{k}$ and $\dot{H}_{i}$ with zero initial value and call them respectively energy, mass and heat fluence. They satisfy the relations

$\mathscr{U}=-\sum_{i} \partial \mathscr{F}_{i} / \partial x_{i}, \quad M^{k}=-\sum_{i} \partial M_{i}^{k} / \partial x_{i}$,

where $x_{i}$ is the cartesian coordinate.

We now introduce arbitrary variations. Replacing differentials by variations in eq. (2.11) and solving for $\delta \mathscr{S}$ we obtain

$\delta \mathscr{S}=(1 / T) \delta \mathscr{U}+\sum_{\rho}\left(A_{\rho} / T\right) \delta \xi_{\rho}-\sum_{k}\left(\phi_{k} / T\right) \delta M^{k}$.

We also write eq. (4.1) in variational form as

$\delta \mathscr{F}_{i}=\sum_{k} \bar{\epsilon}_{k} \delta M_{i}^{k}+\delta H_{i}$.

Hence using (4.2) we obtain

$$
\begin{aligned}
& \delta \mathscr{U}=-\sum_{i} \frac{\partial}{\partial x_{i}}\left(\sum_{k} \bar{\epsilon}_{k} \delta M_{i}^{k}+\delta H_{i}\right), \\
& \delta M^{k}=-\sum_{i} \frac{\partial}{\partial x_{i}} \delta M_{i}^{k} .
\end{aligned}
$$

Substitution of these values into (4.3) yields an expression of the form

$\delta \mathscr{S}=-\sum_{i} \frac{\partial}{\partial x_{i}} \delta S_{i}+\delta s^{*}$,

where

$$
\begin{aligned}
\delta S_{i}= & \sum_{k} \bar{s}_{k} \delta M_{i}^{k}+\delta H_{i} / T, \\
\delta s^{*}= & \sum_{\rho} \frac{A_{\rho}}{T} \delta \xi_{\rho}-\sum_{k i} \frac{\partial}{\partial x_{i}}\left(\frac{\phi_{k}}{T}\right) \delta M_{i}^{k} \\
& +\sum_{i} \frac{1}{\partial x_{i}}\left(\frac{1}{T}\right) \delta \mathscr{F}_{i} .
\end{aligned}
$$


The value (4.6) represents the variation of entropy per unit volume. The first term is due to the entropy flow while $\delta s^{*}$ is the variation of entropy produced per unit volume.

Actually in the applications we use the virtual dissipation

$$
\begin{aligned}
T \delta s^{*}= & \sum_{\rho} A_{\rho} \delta \xi_{\rho}-T \sum_{k i} \frac{\partial}{\partial x_{i}}\left(\frac{\phi_{k}}{T}\right) \delta M_{i}^{k} \\
& -\frac{1}{T} \sum_{i} \frac{\partial T}{\partial x_{i}} \delta \mathscr{F}_{i} .
\end{aligned}
$$

The dissipative forces which generate entropy are

$$
A_{\rho}, \quad-T \frac{\partial}{\partial x_{i}}\left(\frac{\phi_{k}}{T}\right), \quad-\cdots \frac{1}{T} \frac{\partial T}{\partial x_{i}} .
$$

They provide a measure of the departure of the system from reversibility and may be called disequilibrium forces. They are conjugate to the coordinates $\xi_{\rho}, M_{i}^{k}$ and $\mathscr{F}_{i}$.

\section{New expression of the affinity in terms of chemical kinetics}

We may draw an analogy between viscous forces in mechanics and dissipative forces in irreversible thermodynamics. For the case of chemical reactions this analogy has never been formulated in a satisfactory way. For example in a viscous fluid mixture the viscous stress

$\tau_{i j}=\tau_{i j}\left(m_{k}, T, \dot{\epsilon}_{\mu \nu}\right)$

may be expressed as a function of the temperature $T$, the concentrations $m_{k}$ of the constituents and the rate of deformation $\dot{\epsilon}_{\mu \nu}$. This relation embodies the kinetics of the dissipative process. A similar relation for chemical reactions may be derived as follows. We write the rate of reaction $\xi_{\rho}$ and the affinity $A_{\rho}$ as functions of $m_{k}$ and $T$

$\dot{\xi}_{\rho}=f_{\rho}\left(m_{k}, T\right), \quad A_{\rho}=A_{\rho}\left(m_{k}, T\right)$.

Assume that by adding masses $\sum_{\sigma} \nu_{k \sigma} \Delta \xi_{\sigma}$ to $m_{k}$ we reach chemical equilibrium for which $\xi_{\rho}$ and $A_{\rho}$ vanish. Hence we may write (5.2) in the form

$\dot{\xi}_{\rho}=f_{\rho}\left(m_{k}, T\right)-f_{\rho}\left(m_{k}+\sum_{\sigma} \nu_{k o} \Delta \xi_{o}, T\right)$,

$A_{\rho}=A_{\rho}\left(m_{k}, T\right)-A_{\rho}\left(m_{k}+\sum_{\sigma} \nu_{k \sigma} \Delta \xi_{\sigma}, T\right)$.
We solve equations (5.3) for $\Delta \xi_{\sigma}$ and substitute the result into (5.4). This yields

$A_{\rho}=\mathscr{R}_{\rho}\left(m_{k}, T, \xi_{\rho}\right)$,

where the affinity $\mathscr{R}_{\rho}$ is now expressed as a function of $m_{k}, T$ and $\dot{\xi}_{\rho}$. Relation (5.5) is entirely analogous to (5.1) and embodies the chemical kinctics.

In many cases we may approximate expression (5.5) by a linear function of $\xi_{\rho}$ and write

$\mathscr{R}_{\rho}=\sum_{\sigma} \mathscr{B}_{\rho \sigma}\left(m_{k}, T\right) \dot{\xi}_{\sigma}$,

where $\mathscr{B}_{\rho \mathrm{o}}$ are functions of $m_{k}$ and $T$. If in addition Onsager's reciprocity relations are verified we may introduce a dissipation function

$\mathscr{D}^{\text {ch }}=\frac{1}{2} \sum_{\rho \sigma} \mathscr{B}_{\rho \sigma} \dot{\xi}_{\rho} \dot{\xi}_{\sigma}$

and write (5.6) as

$\mathscr{R}_{\rho}=\partial \mathscr{D}^{\mathrm{ch}} / \partial \dot{\xi}_{\rho}$.

Referring to relation (4.9), the virtual dissipation for chemical reactions is

$T \delta s^{* \mathrm{ch}}=\sum_{\rho} \mathscr{R}_{\rho} \delta \xi_{\rho}$

and the corresponding rate of dissipation is the positive expression

$T \dot{S}^{* \text { ch }}=\sum_{\rho} \mathscr{R}_{\rho} \xi_{\rho}>0$.

In the linear case, with a dissipation function (5.7) the rate of dissipation becomes

$T \dot{s}^{* \mathrm{ch}}=\sum_{\rho}\left(\partial \mathscr{D}^{\mathrm{ch}} / \partial \dot{\xi}_{\rho}\right) \dot{\xi}_{\rho}=2 \mathscr{D}^{\mathrm{ch}}>0$,

where $\mathscr{D}^{\text {ch }}$ is a positive definite quadratic form in $\dot{\xi}_{p}$ with coefficients functions of $m_{k}$ and $T$.

\section{Kinetics of thermomolecular diffusion and invariance under translation}

According to (4.9) the virtual dissipation due to thermomolecular diffusion may be written

$T \delta s^{* \mathrm{TM}}=\sum_{k i} \mathscr{R}_{i}^{k} \delta M_{i}^{k}+\sum_{i} \mathscr{R}_{i}^{\mathrm{F}} \delta \mathscr{F}_{i}$, 
where the dissipative forces are expressed as

$$
\begin{aligned}
& -T \frac{\partial}{\partial x_{i}}\left(\frac{\phi_{k}}{T}\right)=\mathscr{R}_{i}^{k}\left(m_{k}, T, \dot{M}_{i}^{k}, \dot{\mathscr{F}}_{i}\right), \\
& -\frac{1}{T}\left(\frac{\partial T}{\partial x_{i}}\right)=\mathscr{R}_{i}^{\mathrm{F}}\left(m_{k}, T, \dot{M}_{i}^{k}, \dot{\mathscr{F}_{i}}\right) .
\end{aligned}
$$

The functions $\mathscr{R}_{i}^{k}$ and $\mathscr{R}_{i}^{\mathrm{F}}$ embody the irreversible kinetics of the thermomolecular diffusion. They are functions of the local state $m_{k}$ and $T$ and the Inass and energy fluxes $\dot{M}_{i}^{k} \dot{\mathscr{F}}_{i}$. In general they will be linear functions of the fluxes and obey Onsager's reciprocity principle. Hence they may be written in the form

$\mathscr{R}_{i}^{k}=\partial \mathscr{D}^{\mathrm{TM}} / \partial \dot{M}_{i}^{k}, \quad \mathscr{R}_{i}^{\mathrm{F}}=\partial \mathscr{D}^{\mathrm{TM}} / \partial \dot{\mathscr{F}}_{i}$,

with a thermomolecular dissipation function

$\mathscr{D}^{\mathrm{TM}}=\frac{1}{2} \sum_{i k} C^{l k} \dot{M}_{i}^{l} \dot{M}_{i}^{k}+\sum_{i k} C^{k \mathrm{~F}} \dot{M}_{i}^{k} \dot{\mathscr{F}}_{i}+\frac{1}{2} \sum_{i} C^{\mathrm{F}_{\dot{F}}} \dot{\mathscr{F}}_{i}$.

It is a positive definite quadratic form in $\dot{M}_{i}^{k}$ and $\dot{\mathscr{F}}_{i}$ with coefficients $C^{i k}\left(m_{k}, T\right), \quad C^{k \mathrm{~F}}\left(m_{k}, T\right)$ $C^{\mathrm{F}}\left(m_{k}, T\right)$ functions of $m_{k}$ and $T$.

In many cases the mixture is a fluid medium with intermolecular diffusion. In that case the dissipation function must be invariant under a uniform translation. A uniform increase of velocity $v_{i}$ adds $m_{k} v_{i}$ to $\dot{M}_{i}^{k}$ and $\mathscr{F}_{v_{i}}^{\mathrm{c}}$ to $\dot{\mathscr{F}}_{i}$, where

$\mathscr{F}^{\mathrm{c}}=\sum_{k} m_{k}\left(\bar{\epsilon}_{k}+h_{p T}^{k}\right)$.

The quantity $\bar{\epsilon}_{k}+h_{p T}^{k}$ is the energy required to inject a unit mass of substance isothermally into the mixture and $h_{p T}^{k}$ is the additional heat to be injected in order to maintain the temperature and pressure constant. A dissipation function which satisfies the condition of invariance under translation is obviously of the form

$$
\begin{aligned}
\mathscr{D}^{\mathrm{TM}}= & \frac{1}{2} \sum_{l k i} C^{l k}\left(\dot{M}_{i}^{l} / m_{l}-\dot{M}_{i}^{k} / m_{k}\right)^{2} \\
& +\frac{1}{2} \sum_{k i} C^{k}\left(\dot{M}_{i}^{k} / m_{k}-\dot{\mathscr{F}}_{i} / \mathscr{F}^{\mathrm{c}}\right)^{2} .
\end{aligned}
$$

That this is the only form satisfying the invariance condition can be seen by adding to (6.7) the terms needed in order to obtain the most general quadratic form in $\dot{M}_{i}^{k}$ and $\dot{\mathscr{F}}_{i}$. These terms are

$\sum_{i k} A_{k}\left(\dot{M}_{i}^{k}\right)^{2}+\sum_{i} \mathscr{B}_{i} \dot{F}_{i}^{2}$

However, they must vanish since they are not invariant under translation.

An invariant expression for $\mathscr{D}^{\mathrm{TM}}$ was also derived earlier using the entropy flux $\dot{S}_{i}$ instead of $\dot{\mathscr{F}}_{i}$ [1].

\section{Principle of virtual dissipation and lagrangian equation of reaction-diffusion}

For reaction-diffusion the principle of virtual dissipation developed earlier in a broader context [1] may be derived as follows. We consider a hypersystem constituted by a primary system, supply cells $\mathrm{C}_{k}$, chemical cells $\mathrm{C}_{\mathrm{ch} \rho}$ and a thermal well TW. The primary system is a continuous system occupying the domain $\Omega$ whose energy and entropy are defined by (3.1).

We consider an arbitrary irreversible evolution of the primary system determined by a time sequence of $\xi_{\rho}, M_{i}^{k}$ and $\mathscr{F}_{i}$. By eqs. (2.12) and (4.2) this also determines the time sequence of $\xi_{\rho}, M^{k}$ and $T$. We freeze the system at any particular time and consider arbitrary variations $\delta \xi_{\rho}, \delta M_{i}^{k}, \delta \mathscr{F}_{i}$ in the vicinity of a frozen state. In this variation no heat or matter is exchanged between the hypersystem and the environment, and the variation is generated by the virtual work $\delta W$ performed by external forces on the hypersystem. The work is the purely mechanical work necessary to inject masses and heat into $\Omega$ as required by the values $\delta M_{i}^{k}$ and $\delta H_{i}$ at the boundary of $\Omega$, using supply cells as a source of matter and TW as a source of heat for mechanical heat pumps.

The variational principle is obtained by writing that the variations obey a virtual conservation of energy of the hypersystem. This is written

$\delta U+T_{0} \delta S_{\mathrm{TW}}=\delta W$.

In this expression $\delta S_{\mathrm{TW}}$ represents the increase of entropy of TW, hence $\delta U+T_{0} \delta S_{\mathrm{TW}}$ is the increase of energy of the hypersystem. By introducing the exergy

$V=U-T_{0} S$ 
(7.1) becomes

$\delta V+T_{0} \delta S^{*}=\delta W$,

where

$\delta S^{*}=\delta S+\delta S_{\mathrm{TW}}$

is the total virtual entropy produced in the hypersystem.

Eq. (7.3) expresses the principle of virtual dissipation. It remains to show how to evaluate $\delta W$ and $\delta S^{*}$ for reaction-diffusion.

We write $\delta W$ as a surface integral extended to the boundary $A$ of the domain $\Omega$ with a unit normal $n_{i}$. This value is

$\delta W=-\int_{A} \sum_{k i}\left[\psi_{k} \delta M_{i}^{k}+(\theta / T) \delta H_{i}\right] n_{i} \mathrm{~d} A$,

where $\theta=T-T_{0}$. The mass injected into $\Omega$ per unit area is $-\sum_{i} \delta M_{i}^{k} n_{i}$. It is extracted from the supply cell at the pressure and temperature $p_{0} T_{0}$, then heated and compressed to a suitable pressure and temperature $p_{k}$ and $T$, then injected reversibly into $\Omega$ at the boundary. The purely mechanical work to accomplish this, including the use of heat pumps, per unit mass, was called thermobaric potential [1]. Its value was found to be

$\psi_{k}=\int_{p_{0} T_{0}}^{p_{k} T}\left(\mathrm{~d} p_{k}^{\prime} / \rho_{k}^{\prime}+\theta^{\prime} \mathrm{d} \bar{s}_{k}^{\prime}\right)=\bar{\epsilon}_{k}-T_{0} \bar{s}_{k}$.

The path of integration is arbitrary and corresponds to what we have called a thermobaric transfer. The values of the pressure, density, specific entropy, and temperature along the path are $p_{k}^{\prime}, \rho_{k}^{\prime}, \bar{s}_{k}^{\prime}$ and $T^{\prime}$ with $\theta^{\prime}=T^{\prime}-T_{0}$. In (7.5) the term $(-\theta / T) \delta H_{i} n_{i}$ is the work of a heat pump extracting heat from TW and injecting the heat $\delta H_{i} n_{i}$ through the boundary at the temperature $T=T_{0}+\theta$.

Substitution in (7.5) of the value (7.6) of $\psi_{k}$ and the value $\delta H_{i}$ extracted from (4.4) yields

$$
\begin{aligned}
\delta W= & -\int_{A} \sum_{i}\left[\left(T_{0} / T\right) \sum_{k} \phi_{k} \delta M_{i}^{k}+(\theta / T) \delta \mathscr{F}_{i}\right] \\
& \times n_{i} \mathrm{~d} A .
\end{aligned}
$$

The virtual dissipation is

$$
T_{0} \delta S^{*}=T_{0} \int_{\Omega}\left(\delta s^{* \mathrm{ch}}+\delta s^{* \mathrm{TM}}\right) \mathrm{d} \Omega
$$

where $\delta s^{* \text { ch }}+\delta s^{* T M}$ is the total virtual entropy produced per unit volume. Using the values (5.9) and (6.1) and the values (6.4) for $\mathscr{R}_{i}^{k}$ and $\mathscr{R}_{i}^{\mathrm{F}}$ we obtain

$$
\begin{aligned}
T_{0} \delta S^{*}= & T_{0} \int_{\Omega} \frac{1}{T}\left(\sum_{\rho} \mathscr{R}_{\rho} \delta \xi_{\rho}+\sum_{k i} \frac{\partial \mathscr{D}^{\mathrm{TM}}}{\partial \dot{M}_{i}^{k}} \delta M_{i}^{k}\right. \\
& \left.+\sum_{i} \frac{\partial \mathscr{D}^{\mathrm{TM}}}{\partial \dot{\mathscr{F}}_{i}} \delta \mathscr{F}_{i}\right) \mathrm{d} \Omega .
\end{aligned}
$$

Applying the principle of virtual dissipation (7.3) we may derive the evolution of a reaction-diffusion field in terms of generalized coordinates by proceeding as follows. The reaction-diffusion field is given by the vector fields $\mathscr{F}_{i}$ and $M_{i}^{k}$ and the scalar fields $\xi_{\rho}$. They may be expressed in the form

$\mathscr{F}_{j}=\sum_{i} u_{i j}^{\mathrm{F}} q_{i}, \quad M_{j}^{k}=\sum_{i} u_{i j}^{k} q_{i}, \quad \xi_{\rho}=\sum_{i} u_{i}^{\rho} q_{i},(7.10)$

where $u_{i j}^{\mathrm{F}}\left(x_{l}\right), u_{i j}^{k}\left(x_{l}\right), u_{i}^{\rho}\left(x_{l}\right)$ are suitable chosen functions of the coordinates $x_{l}$ while $q_{i}$ are generalized coordinates to be determined as functions of time. Once we know $q_{i}$ we obtain from (4.2)

$$
\begin{gathered}
\mathscr{U}=-\sum_{j} \partial \mathscr{F}_{j} / \partial x_{j}=\sum_{i} w_{i}^{\mathrm{F}} q_{i}, \\
M^{k}=-\sum_{j} \partial M_{j}^{k} / \partial x_{j}=\sum_{i} w_{i}^{k} q_{i},
\end{gathered}
$$

where

$$
w_{i}^{\mathrm{F}}=-\sum_{j} \partial u_{i j}^{\mathrm{F}} / \partial x_{j}, \quad w_{i}^{k}=-\sum_{j} \partial u_{i j}^{k} / \partial x_{j} .
$$

Knowing $\mathscr{U}, M^{k}$ and $\xi_{\rho}$ we determine the temperature $T$ from relations (2.12).

The generalized coordinates as functions of time are governed by a system of differential equations called lagrangian equations. They are obtained from the variational principle (7.3) by introducing arbitrary variations $\delta q_{i}$ in the values (7.10). The exergy (7.2) may be considered as a function $V\left(q_{i}\right)$ of the generalized coordinates $q_{i}$. We may write

$$
\begin{aligned}
& \delta V=\sum_{i}\left(\partial V / \partial q_{i}\right) \delta q_{i}, \quad T_{0} \delta S^{*}=\sum_{i} R_{i} \delta q_{i}, \\
& \delta W=\sum_{i} Q_{i} \delta q_{i} .
\end{aligned}
$$


Substituting the values (7.13) into the variational principle (7.3) with arbitrary variations $\delta q_{i}$ yields the lagrangian equations of reaction-diffusion as

$\partial V / \partial q_{i}+R_{i}=Q_{i}$.

It is easily shown that $\partial V / \partial q_{i}$ may be obtained without actually evaluating $V\left(q_{i}\right)$ as an explicit function of $q_{i}$. The generalized driving force $Q_{i}(t)$ is a given function of time if the values $\phi_{k}$ and the temperature $T$ are determined as functions of time at the boundary. The combined generalized thermomolecular and chemical dissipative force

$R_{i}=R_{i}\left(q_{l}, \dot{q}_{l}\right)$

is a function of $q_{l}$ and $\dot{q}_{l}$ with the fundamental property

$\sum R_{i} \dot{q}_{i}>0$.

\section{New stability criteria of evolution}

Consider a systcm where the values $\phi_{k}$ and the temperature $T$ are time independent at the boundary. A possible evolution of the system is a steady state where the temperature $T$ and the masses $m_{k}$ per unit volume are also time independent along with the time derivatives $\dot{\mathscr{F}}_{i}, \dot{M}_{i}^{k}$ and $\dot{\xi}_{\rho}$ throughout the system.

The question is to evaluate the stability of such a steady state far from equilibrium. The lagrangian equations (7.14) provide a completely general tool for such a stability analysis. The steady state field may be represented by putting

$q_{1}=\alpha t, \quad q_{2}=q_{3}, \ldots, q_{n}=0$

in expressions (7.10) where the steady state corresponds to the value $q_{1}=\alpha t$ proportional to the time $t$. A perturbed field is represented in terms of small perturbations $\Delta q_{i}$ by putting

$q_{1}=\alpha t, \quad q_{2}=\Delta q_{2}, \quad q_{3}=\Delta q_{3}, \ldots, q_{n}=\Delta q_{n}$.

If the values of the temperature and $\phi_{k}$ remain unperturbed at the boundary, $Q_{i}$ remains unperturbed. In that case the lagrangian eqations (7.14) for small perturbations become

$$
\sum_{j}\left(\partial^{2} V / \partial q_{i} \partial q_{j}\right) \Delta q_{j}+\Delta R_{i}=0
$$

where

$$
\begin{gathered}
\Delta R_{i}=\sum_{j}\left(\partial R_{i} / \partial \dot{q}_{j}\right) \Delta \dot{q}_{j}+\sum_{j}\left(\partial R_{i} / \partial q_{j}\right) \Delta q_{j}, \\
j=2,3, \ldots, n .
\end{gathered}
$$

Putting

$$
\begin{aligned}
& \partial^{2} V / \partial q_{i} \partial q_{j}=a_{i j}=a_{j i}, \\
& \quad c_{i j}=\partial R_{i} / \partial q_{j}, \quad r_{i j}=\partial R_{i} / \partial \dot{q}_{j},
\end{aligned}
$$

the perturbation equations (8.2) are written

$$
\sum_{j}\left(a_{i j}+c_{i j}\right) \Delta q_{j}+\sum_{j} r_{i j} \Delta \dot{q}_{j}=0 .
$$

The coefficients (8.4) are evaluated for unperturbed values (8.1) of $q_{i}$ and it is easily seen that they are constants for a steady state.

The stability analysis of the steady state reaction-diffusion in the general case is thus reduced to solving a system of homogeneous differential equations with constant coefficients. The system will be stable if all characteristic roots have negative real parts.

There is, however, a particular case where it is possible to take advantage of the thermodynamic properties to derive a direct stability criterion. Let us assume that the affinities are linear functions of the reaction rates, i.e.

$\mathscr{R}_{\rho}=\sum_{\sigma} \mathscr{B}_{\rho \sigma}\left(m_{k}, T\right) \dot{\xi}_{\sigma}$.

Since the thermomolecular dissipative forces (6.4) are linear functions of the rates $\dot{\mathscr{F}}_{i}$ and $\dot{M}_{i}^{k}$, the total dissipative generalized forces $R_{i}$ are also linear functions of $\dot{q}_{i}$. Hence we may write

$R_{i}=\sum_{j} r_{i j}\left(q_{l}\right) \dot{q}_{j}$

where the coefficients $r_{i j}$ are functions of the generalized coordinates $q_{l}$. An important property here is the positive-definiteness (7.16) of

$\sum_{i} R_{i} \dot{q}_{i}=\sum_{i j} r_{i j}\left(q_{i}\right) \dot{q}_{i} \dot{q}_{j}$

In addition we shall also assume that $\mathscr{R}_{\rho}$ is expressed by (5.8). Due to expressions (6.4) for $\mathscr{R}_{i}^{k}$ and $\mathscr{R}_{i}^{\mathrm{F}}$ this implies $r_{i j}=r_{j i}$. 
In the present case the perturbed value (8.3) of $R_{i}$ becomes

$\Delta R_{i}=\sum_{j}\left(\partial r_{i 1} / \partial q_{j}\right) \dot{q}_{1} \Delta q_{j}+\sum_{j} r_{i j}\left(q_{l}\right) \Delta \dot{q}_{j}$.

Hence

$c_{i j}=\left(\partial r_{i 1} / \partial q_{j}\right) \dot{q}_{1}$,

while $r_{i j}$ is a constant obtained by substituting for $q_{l}$ the values $q_{1}$ and $q_{2}=q_{3}, \ldots, q_{n}=0$. According to (8.8) the coefficients $r_{i j}$ are positive-definite i.e $\sum_{i j} r_{i j} \Delta q_{i} \Delta q_{j}>0$.

This important relation based on irreversible thermodynamics leads to a new stability criterion obtained as follows. Multiply eqs. (8.5) by $\Delta q_{i}$ and add the results. We obtain

$\sum_{i j}\left(a_{i j}+c_{i j}\right) \Delta q_{i} \Delta q_{j}+\sum_{i j} r_{i j} \Delta q_{i} \Delta \dot{q}_{j}=0$.

This may be written

$\sum_{i j}\left(a_{i j}+c_{i j}\right) \Delta q_{i} \Delta q_{j}+\frac{1}{2} \mathrm{~d} D^{\prime} / \mathrm{d} t=0$,

where

$D^{\prime}=\sum_{i j} r_{i j} \Delta q_{i} \Delta q_{j}$

According to (8.11) it is positive-definite. Let us assume that

$\sum_{i j}\left(a_{i j}+c_{i j}\right) \Delta q_{i} \Delta q_{j}>0$,

i.e. that it is also positive-definite. In that case eq. (8.13) shows that $\mathrm{d} D^{\prime} / \mathrm{d} t<0$. Since $D^{\prime}$ is positive-definite it means that the perturbations $\Delta q_{i}$ tend to zero. As a consequence condition (8.15) is a sufficient condition for the steady state to be stable.

If condition (8.15) is not fulfilled the perturbations may be unstable and contain exponentially increasing solutions. Whether the perturbed solution is stable or unstable two cases may be distinguished.

(1) If $c_{i j}=c_{j i}$ all characteristic roots are real and the perturbations are non-oscillatory.

(2) If $c_{i j} \neq c_{j i}$ some of the perturbation terms may be oscillatory.

An interesting stability criterion may also be derived under fixed boundary conditions by multiplying eqs. (8.2) by $\Delta \dot{q}_{i}$ and adding the results. We obtain

$\sum_{i j}\left(\partial^{2} V / \partial q_{i} \partial q_{j}\right) \Delta \dot{q}_{i} \Delta q_{j}+\sum_{i} R_{i} \Delta \dot{q}_{i}=0$.

If we assume

$\sum_{i} R_{i} \Delta \dot{q}_{i}>0$

and

$\sum_{i j}\left(\partial^{2} V / \partial q_{i} \partial q_{j}\right) \Delta q_{i} \Delta q_{j}>0$

by an argument similar to that used above for equation (8.12) we conclude that $\Delta q_{i}$ must tend to zero. Hence when the two inequalities (8.17) and (8.18) are verified simultaneously the steady state evolution is stable. Condition (8.17) is similar to the one derived by Glansdorff and Prigogine [2]. However, it is obtained here by a very simple and general direct procedure. The additional condition (8.18) required may be considered as expressing in precise form the assumption of so-called "local equilibrium" implicit in current procedures.

\section{References}

[1] M.A. Biot, in: Advances in applied mechanics, Vol. 24. New variational-lagrangian irreversible thermodynamics with application to viscous flow, reaction-diffusion and solid mechanics (Wilpy, New York, 1984) pp. 1-91.

[2] P. Glansdorff and I. Prigogine, Structure, stabilité et fluctuations (Masson. Paris, 1971).

$$
\text { Academic Press }
$$

